www.jmscr.igmpublication.org

Impact Factor (SJIF): 6.379

Index Copernicus Value: 79.54

ISSN (e)-2347-176x ISSN (p) 2455-0450

crossrefDOI: https://dx.doi.org/10.18535/jmscr/v6i10.176

Journal Of Medical Science And Clinical Research

\title{
A Case Study of Rifampicin Resistance in Newly Detected Sputum Negative Pulmonary Tuberculosis Patients by CBNAAT
}

\author{
Authors

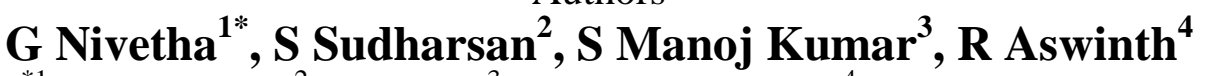 \\ ${ }^{* 1}$ Post graduate, ${ }^{2}$ Professor, ${ }^{3}$ Assistant Professor, ${ }^{4}$ Assistant Professor \\ Department of General Medicine, Rajah Muthiah Medical College and Hospital (RMMC\&H), \\ Chidambaram, Tamil Nadu, India \\ Corresponding Author
}

Dr G. Nivetha

Email:nivecoolz@yahoo.com

\begin{abstract}
Background: Tuberculosis is the most common communicable disease causing morbidity and mortality worldwide. India is the country with the highest burden of MDR-TB. The WHO has endorsed CBNAAT in December 2010, an automated real time PCR based rapid diagnostic test, simultaneously detecting mycobacterium tuberculosis and rifampicin resistance within two hours. ${ }^{1,2,3}$ WHO has recommended in 2013, the use CBNAAT in sputum AFB negative pulmonary tuberculosis, extra pulmonary tuberculosis, paediatric tuberculosis and in immune compromised patients like HIV and diabetes mellitus. ${ }^{4,5,6}$ Various studies shows the prevalence of MDR/RR TB in previously treated patients.

Aim of the Study: This study was done to detect the rifampicin resistance in newly detected sputum AFB negative pulmonary tuberculosis patient through CBNAAT.

Material and Methods: This study includes 140 cases of Presumptive pulmonary tuberculosis patients who were smear negative during the period during the period between Nov 2016 to April 2018 in all presumptive pulmonary tuberculosis patients who attended OP or got admitted in Rajah Muthiah medical college and hospital, Chidambaram. These patients were enrolled and CBNAAT was provided.

Results: Out of these 140 cases, MTB was detected in 54 cases (38.6\%) and in rest of the 86 cases MTB not detected. Among 54 cases, 2 were rifampicin resistance and rest of them were rifampicin sensitive. These 2 rifampicin resistance was diagnosed in newly detected pulmonary tuberculosis patients. This study shows the prevalence of $3.7 \%$ MDR-TB in primary pulmonary tuberculosis.

Conclusion: This study shows that CBNAAT is more superior to sputum microscopy by detecting additional pulmonary tuberculosis cases and drug resistance which are missed by sputum AFB stain within few hours. We concluded that all sputum AFB negative presumptive pulmonary tuberculosis patients should undergo novel technique like CBNAAT and LPA to diagnose the undetected TB and DR - TB.

Keywords: Sputum AFB negative, CBNAAT, New case, Rifampicin resistance.
\end{abstract}

\section{Introduction}

Tuberculosis is the oldest disease caused by mycobacterium tuberculosis transmitted from infectious pulmonary tuberculosis patients through the airborne spread of droplet nuclei, most often affecting the lungs. It still remains the one of 
the commonest infectious cause of death in developing countries. According to the WHO report in 2016, 10.4 million cases developed TB and 1.7 million people died from the disease including 400,000 people who are co infected with HIV. Out of 10.4million cases, 490,000 incident cases of MDR/RR - TB are reported. Out of 10.4 milllion cases, only 6.3 million cases were detected. Remaining 4million cases were not detected. Among 10.4 million cases, 2.79 million cases were reported from India. India constitutes about $27 \%$ of global TB burden. ${ }^{8}$ India ranked the top of seven globally TB burden countries followed by Indonesia, China, Philippines, Pakistan, South Africa and Nigeria. Disease caused by mycobacterium tuberculosis which is resistant to at least Isoniazid (H) and/or Rifampicin (R) with or without other first line antituberculosis drugs is defined as MDR/RR TB. About $47 \%$ of MDR/RR-TB was in India, China and Russia. Out of 2.79million Indian cases, 147000 cases were MDR/RR -TB and 87000 were co infected with HIV. Overall mortality rate was $15.6 \%$ in India in $2016 .^{7}$

Smear microscopy by Ziehl Neelsen staining which is the cornerstone for the diagnosis of tuberculosis is very rapid, cheap and easy which requires at least 10,000 bacilli per $\mathrm{ml}$ of sputum to make the diagnosis of tuberculosis. It has a specificity of $99 \%$ and sensitivity of only $20-$ $80 \% .{ }^{9}$ Major disadvantage of smear microscopy is that it fails to detect the rifampicin resistance. Gold standard technique is sputum culture which takes about $6-8$ weeks for growth and has sensitivity of $80-85 \%$ and specificity of 98\%.where liquid culture (MGIT) require 421days for growth of at least 10-100 tuberculosis bacilli/ml. Drug susceptibility takes 1528days. ${ }^{10}$ MDR TB and XDR TB should be confirmed by culture. Major drawback of culture is time lag for initiation of appropriate treatment. Drug resistance is the major challenge to end TB. Emergence of MDR -TB is not due to single factor. ${ }^{11}$ Majority of active pulmonary tuberculosis patients manifest as sputum AFB negative.
Mainstay of Tuberculosis management is early diagnosis and appropriate treatment thereby reducing the chance of transmission. To overcome all these drawbacks, the WHO has endorsed a newer rapid diagnostics technique like Line probe assay (manual) and automated technique like CBNAAT detecting DR - TB within two days and two hours respectively. CBNAAT (Cartridge based nucleic acid amplification test) which was recommended as add on test for sputum smear negative specimens and first line diagnostic test in MDR/RR - TB and HIV associated TB.

GENE XPERT (MTB/RIF - assay) recommended in 2010 as an automated hemi nested real time PCR and reverse transcriptase PCR based diagnostic tests detects both mycobacterium tuberculosis complex DNA and rifampicin resistance using 5 molecular beacons(A-E)within 2 hours. ${ }^{2}$ G4 version has been recommended by the WHO from 2013 designed by Cepheid, Sunnyvale, CANADA. ${ }^{12}$ It helps the physician to make expert decision in undetected cases from smear microscopy in presumptive pulmonary tuberculosis patients and to interrupt the transmission in drug resistant strains and start the appropriate treatment without any time lag on the same visit. It helps to avoid injudicious use of ATT in the better interest of patients. CBNAAT assay is performed by sample processing, DNA extraction, nucleic acid amplification and detects the target sequences and mutation in rpo gene which codes for rifampicin resistance. ${ }^{1,13,14}$ CBNAAT has sensitivity of $99 \%$ and $>80 \%$ in smear positive and smear negative cases respectively. It also has sensitivity of $95.1 \%$ and specificity $98.4 \%$ in detecting rifampicin resistance. $^{15}$

\section{Material and Methods}

This study was conducted at Rajah Muthiah Medical College and Hospital, Chidambaram, during the period between Nov 2016 to April 2018 in all presumptive pulmonary tuberculosis patients who attended OP or got admitted in our hospital. Inclusion criteria include patients above 
14yrs of age with sputum smear Negative, Patients co infected with HIV, Microbiologically confirmed retreatment patients(recurrent, treatment after loss of follow up and failure cases). Exclusion criteria includes smear positive patients, extra pulmonary tuberculosis patients and patients currently on ATT drugs. Patient's demographic data was noted. Detailed history, clinical examination, routine investigation, chest $\mathrm{X}$ ray, sputum smears AFB staining was done. 140 Sputum negative patients were evaluated in this study and sputum sample has been sent for CBNAAT. This study was done to evaluate the role of CBNAAT in diagnosing pulmonary tuberculosis and rifampicin resistance in sputum smear negative patients. The statistical analysis was done with Pearson Chi Square test.

Sputum sample should be collected in falcon tube are mixed with sample reagent (sodium hydroxide and isopropanol) in the ratio of 2:1.Sample is mixed well and incubated for $15 \mathrm{mins}$ at room temperature. The treated sample is transferred into the single use plastic cartridge which is preloaded with liquid buffers and lyophilized reagent beads required for sample processing. The CBNAAT assay is fully automated with d"38 cycles threshold. It detects mycobacterium tuberculosis when at least two of the five microbe probes show positive signals and also states rifampicin resistance when $\ddot{A C T}>3.5$ cycles. ${ }^{1,16,17}$ Results of CBNAAT obtained as: MTB not detected, MTB detected rifampicin resistance detected, MTB detected Rifampicin resistance not detected, invalid and indeterminate. ${ }^{4,19}$

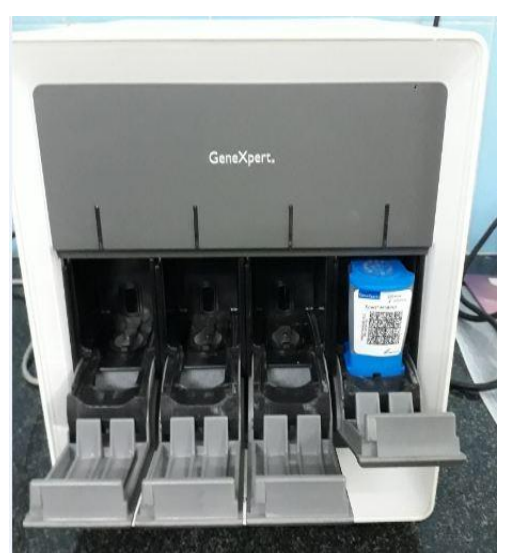

Gene Xpert

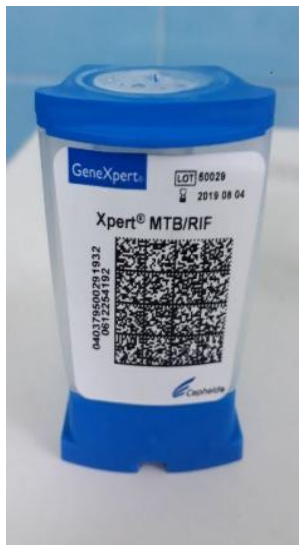

Cartridge

\section{Observations and Results}

Table 1: Age vs Sex Distribution

\begin{tabular}{|l|c|c|c|c|}
\hline Age group in yrs & Female & Male & Total & Percentage \\
\hline$<20$ & 1 & 3 & 4 & $2.9 \%$ \\
\hline $21-30$ & 8 & 6 & 14 & $10.0 \%$ \\
\hline $31-40$ & 6 & 11 & 17 & $12.7 \%$ \\
\hline $41-50$ & 11 & 19 & 30 & $21.4 \%$ \\
\hline $51-60$ & 7 & 27 & 34 & $24.3 \%$ \\
\hline $61-70$ & 7 & 21 & 28 & $20.0 \%$ \\
\hline$>71$ & 2 & 11 & 13 & $9.3 \%$ \\
\hline Total & 42 & 98 & 140 & $100 \%$ \\
\hline
\end{tabular}

Graph 1: Age vs Sex Distribution

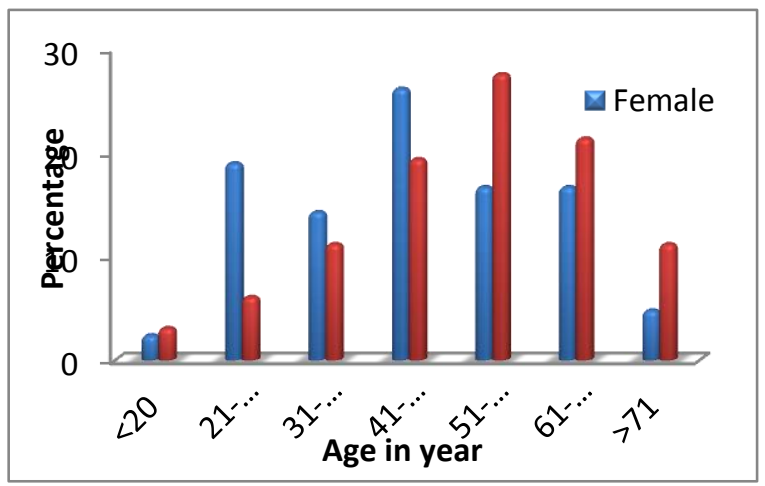




\section{JMSCR Vol||06||Issue||10||Page 1062-1072||October}

34 patients are in 51 to 60 yrs followed by 30 were in 41-50yrs and 28 were in 61-70yrs of age group are most commonly involved age groups in this study . Only 4 patients are below 20 yrs of age group which is least commonly involved. Other age groups like 31-40yrs, 21-30 yrs and above $71 \mathrm{yrs}$ are moderately involved in the frequency of 17,14 and 13 respectively.

Table 2: Sex Distribution

\begin{tabular}{|l|c|c|}
\hline Sex & Total & Percentage \\
\hline Male & 98 & $70 \%$ \\
\hline Female & 42 & $30 \%$ \\
\hline Total & 140 & $100 \%$ \\
\hline
\end{tabular}

Graph 2: Sex Distribution

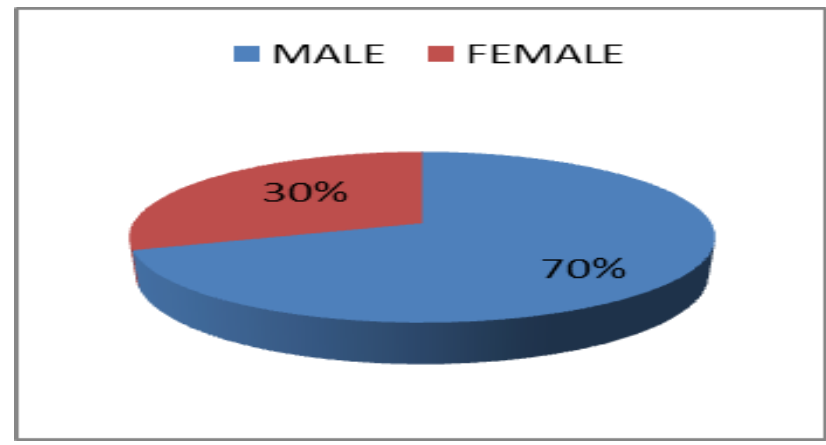

In our study, out of 140 smear negative pulmonary Mohanty et al and Ishwarappagol et al of male tuberculosis patients, 98 patients were males and predominance.

remaining 42 were female which correlates with

Table 3: Clinical Features of Smear Negative Presumptive Pulmonary Tuberculosis

\begin{tabular}{|l|c|c|}
\hline Symptoms & No. of Cases & $\%$ \\
\hline Cough with expectoration & 133 & $95 \%$ \\
\hline Fever & 90 & $64.2 \%$ \\
\hline Breathlessness & 99 & $70.7 \%$ \\
\hline Chest pain & 44 & $31.4 \%$ \\
\hline Hemoptysis & 28 & $20 \%$ \\
\hline Wheeze & 18 & $12.9 \%$ \\
\hline
\end{tabular}

Majority of patients (133) presented with cough with expectoration predominantly followed by 99 (70.7\%) of them presented with breathlessness and 90 patients $(64.2 \%)$ presented with fever in this study which is similar to France Le Palud et al study (2014).

Table 4: Diabetic status

\begin{tabular}{|l|l|l|}
\hline & Total & \% \\
\hline Diabetic & 46 & $32.9 \%$ \\
\hline Non diabetic & 94 & $67.1 \%$ \\
\hline Total & 140 & $100 \%$ \\
\hline
\end{tabular}

Only 46 patients $(32.9 \%)$ were diabetic in this study population of 140 which similar to pradeep Ishwarappagol et al study (28.6\%). Rest of the study population about $94(67.1 \%)$ were non diabetic. 
Table 5: Risk factor distribution in smear negative presumptive pulmonary tuberculosis patients

\begin{tabular}{|l|c|c|}
\hline Risk Factor & No. of Cases & Percentage\% \\
\hline Smoking & 80 & $57.1 \%$ \\
\hline Alcohol & 53 & $37.9 \%$ \\
\hline Contact history & 37 & $26.4 \%$ \\
\hline Past h/o PTB & 38 & $27.1 \%$ \\
\hline
\end{tabular}

In this study, 80 out of 140 patients were smokers, H/O PTB and 37 had Contact history constitutes being the major risk factor $(57.9 \%)$ followed by about $27.1 \%$ and $26.4 \%$ respectively. 53 patients were alcoholics.38 Patients had Past

Table 6: Treatment History of Past PTB

\begin{tabular}{|l|c|c|c|}
\hline Past H/O PTB & No.of cases & Percentage\% & Incidence of PTB \\
\hline Treatment after loss to follow up & 18 & $47.4 \%$ & 10 \\
\hline Completed & 20 & $52.6 \%$ & 4 \\
\hline Total & 38 & $100 \%$ & 14 \\
\hline
\end{tabular}

Out of 38 patients with past h/o PTB, 18(47.6\%) Among these 18, 10 of them developed were Treatment after loss to follow up and remaining 20 patients completed the treatment. reactivation and among 20 who completed their retreatment had recurrence.

Graph 3: Treatment History of Past PTB

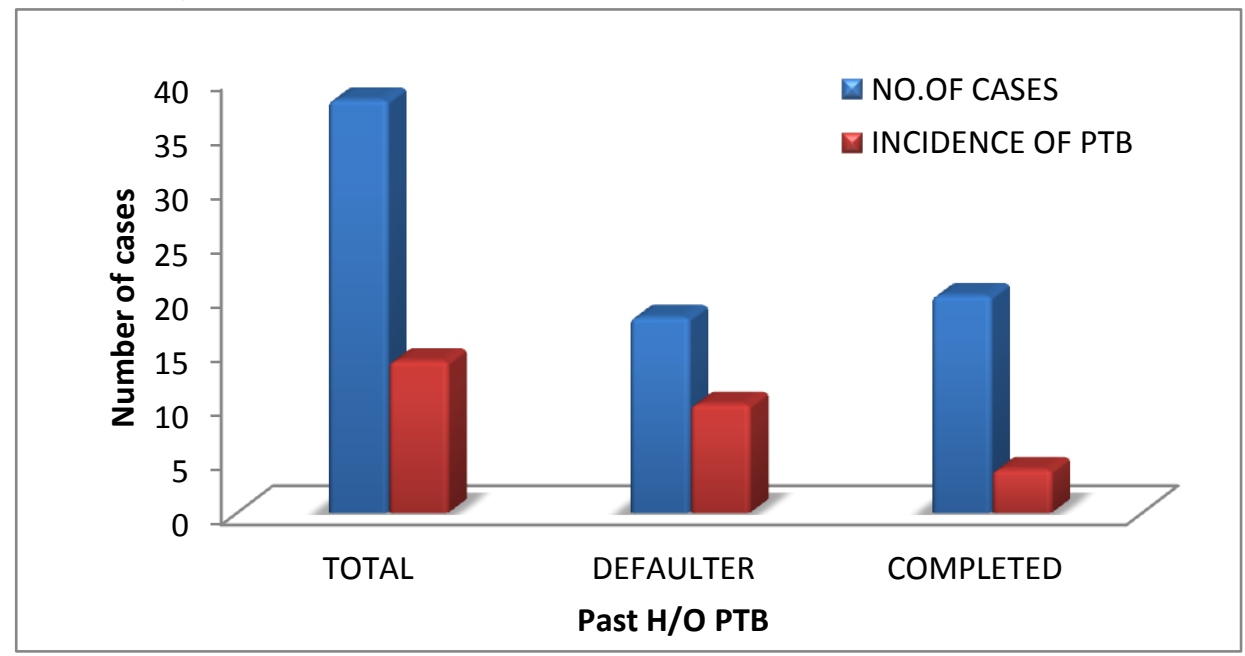

Table 7: Chest X-ray Finding

\begin{tabular}{|l|c|c|c|c|}
\hline Chest xray finding & $\begin{array}{c}\text { No. Of } \\
\text { cases }\end{array}$ & Percentage & $\begin{array}{c}\text { CBNAAT } \\
\text { Positive }\end{array}$ & $\begin{array}{c}\text { CBNAAT } \\
\text { Negative }\end{array}$ \\
\hline Infiltrate & 26 & $18.6 \%$ & 15 & 11 \\
\hline Patchy infiltrate & 10 & $7.1 \%$ & 3 & 7 \\
\hline Consolidation & 29 & $20.7 \%$ & 13 & 16 \\
\hline Nodular opacity & 8 & $5.7 \%$ & 2 & 6 \\
\hline Fibrocavity & 30 & $21.4 \%$ & 20 & 10 \\
\hline Bronchectactic changes & 8 & $5.7 \%$ & 0 & 8 \\
\hline Normal study & 17 & $12.1 \%$ & 1 & 16 \\
\hline Other abnormalities & 10 & $7.1 \%$ & 0 & 10 \\
\hline Thick wall cavity & 2 & $1.4 \%$ & 0 & 2 \\
\hline Total & 140 & $100 \%$ & 54 & 86 \\
\hline
\end{tabular}


Graph 4: Chest X-ray finding in CBNAAT positive and negative cases

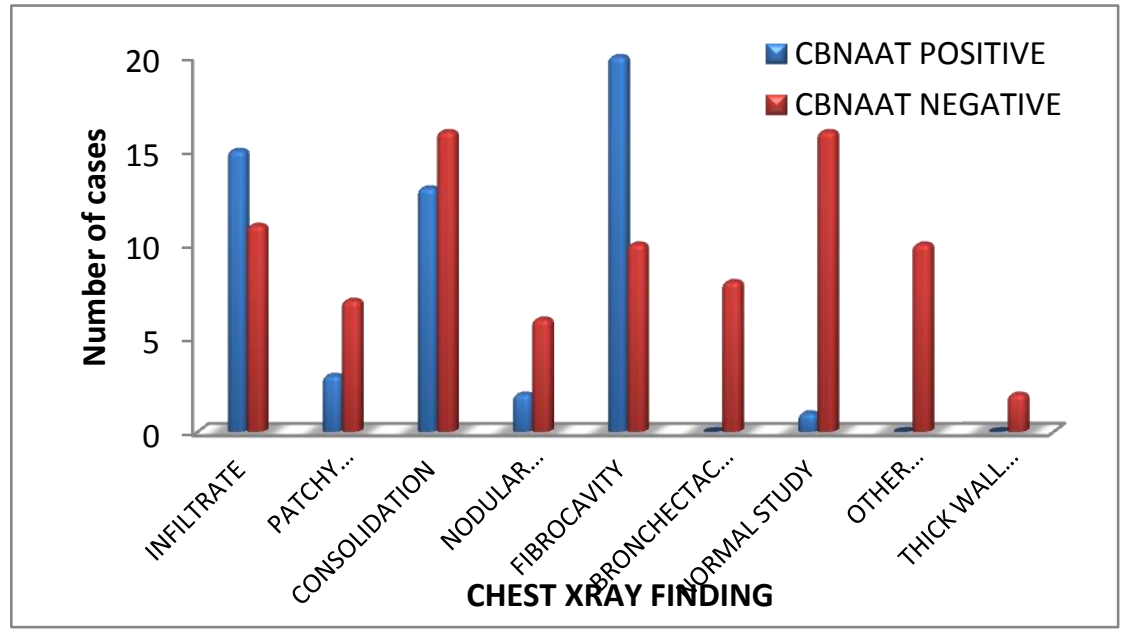

In this study,36 patients had radiological finding of Infiltrate including patchy infiltrate is the most common radiological finding reported followed by 30 had fibro cavity lesion and 29 patients had consolidation. In CBNAAT positive cases, fibro cavity and infiltrate being predominate, whereas consolidation in CBNAAT negative cases which is same line with Poojan shretha et al study with infiltrate predominate in CBNAAT positive cases.

Table 8: Chest X Ray Involvement

\begin{tabular}{|l|c|c|}
\hline Xray involvement & No. Of cases & Percentage \\
\hline UZ & 58 & $41.4 \%$ \\
\hline MZ & 5 & $3.6 \%$ \\
\hline LZ & 31 & $22.1 \%$ \\
\hline UZ+MZ & 2 & $1.4 \%$ \\
\hline MZ+LZ & 11 & $7.9 \%$ \\
\hline All zone & 16 & $11.4 \%$ \\
\hline Normal & 17 & $12.1 \%$ \\
\hline Total & 140 & $100 \%$ \\
\hline
\end{tabular}

76 patients of 140 cases presented with upper zone zone involvement and less frequently middle zone involvement predominately followed by lower are involved.

Table 9: Chest X-ray Side Involvement

\begin{tabular}{|l|c|}
\hline Chest x-ray side involvement & No.of cases \\
\hline Right & 67 \\
\hline Left & 34 \\
\hline Bilateral & 22 \\
\hline Normal & 17 \\
\hline
\end{tabular}

Graph 5: Chest X-ray Side Involvement

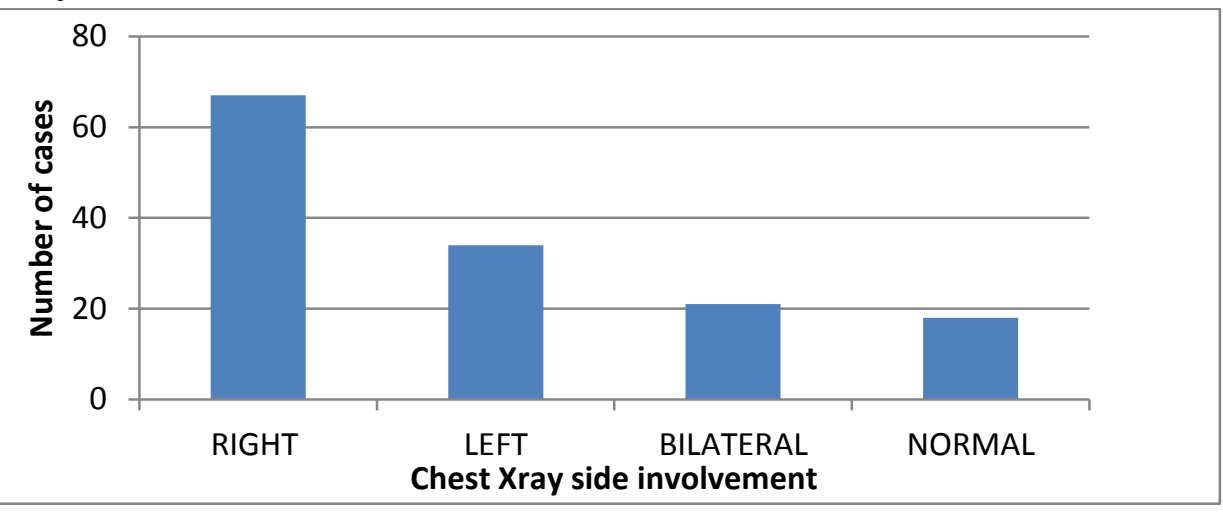




\section{JMSCR Vol||06||Issue||10||Page 1062-1072||October}

Right sided radiological finding are most commonly involved in this study. About 67 cases reported to have right sided involvement. 17 of them reported to have normal chest $\mathrm{X}$ ray. Rest left sided and bilateral involvement have more or else equal distribution in 34 and 22 cases respectively.

Table 10: CBNAAT Positive vs Negative Distribution

\begin{tabular}{|l|c|c|}
\hline CBNAAT Positive & 54 & $38.6 \%$ \\
\hline CBNAAT Negative & 86 & $61.4 \%$ \\
\hline Total & 140 & $100 \%$ \\
\hline
\end{tabular}

Graph 6: CBNAAT Positive vs Negative Distribution

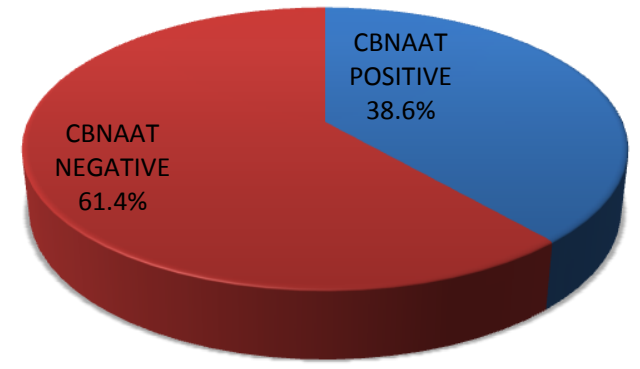

Table 11: Level of MTB Detection by CBNAAT

\begin{tabular}{|l|c|c|}
\hline & Total & Percentage \\
\hline No MTB & 86 & 61.4 \\
\hline Very low & 4 & 2.9 \\
\hline Low & 17 & 12.1 \\
\hline Medium & 24 & 17.1 \\
\hline High & 9 & 6.4 \\
\hline Total & 140 & 100.0 \\
\hline
\end{tabular}

Out of 54 CBNAAT positive cases, majority of cases, low level in 17 cases, 9 of them as High cases detected as medium level of MTB in 24
Out of 140 cases, 98 cases were reported to be CBNAAT positive and rest of the 86 patients were reported as CBNAAT negative. Among these 86 cases, 2 of them were started on ATT based on their Xray finding. These 2 patients who reported as CBNAAT negative may due to paucibacillary, inadequate sputum sample and small lung lesion. $38.6 \%$ of CBNAAT positivity correlates with other Pradeep Ishwarappagol et al study of $36.3 \%$ and $37.5 \%$ of Sowjanya et al. ${ }^{17,18}$

Graph 7: Level of MTB Detection by CBNAAT

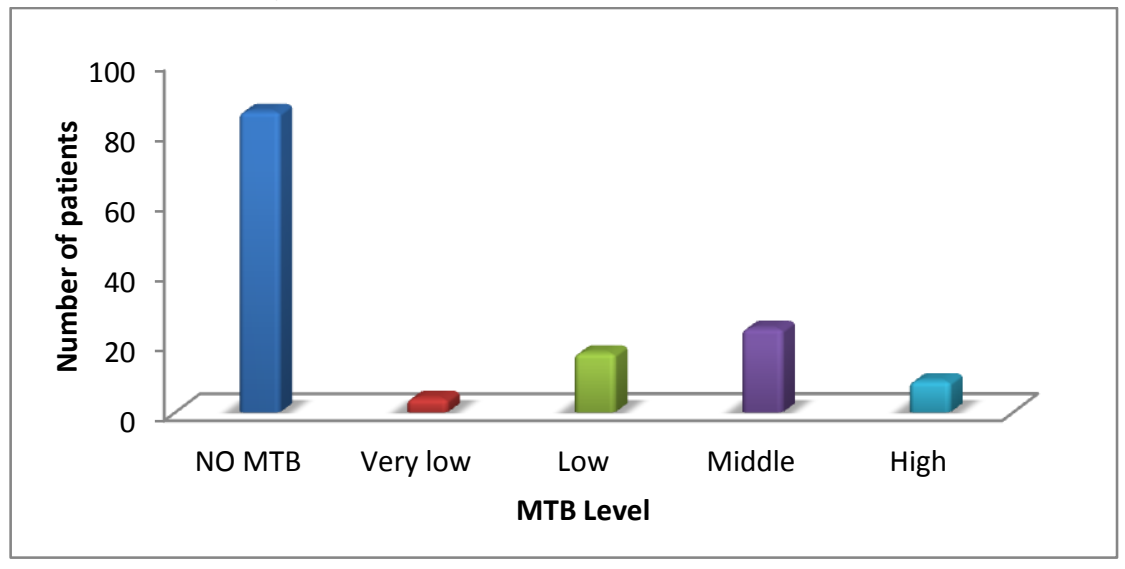


Table 12: Rifampicin Resistance Detection

\begin{tabular}{|l|c|c|}
\hline Rifampicin resistance & No.of cases & Percentage \\
\hline Detected & 2 & $3.7 \%$ \\
\hline Not detected & 52 & $96.3 \%$ \\
\hline & 54 & $100 \%$ \\
\hline
\end{tabular}

Graph 8: Rifampicin Resistnace Distribution

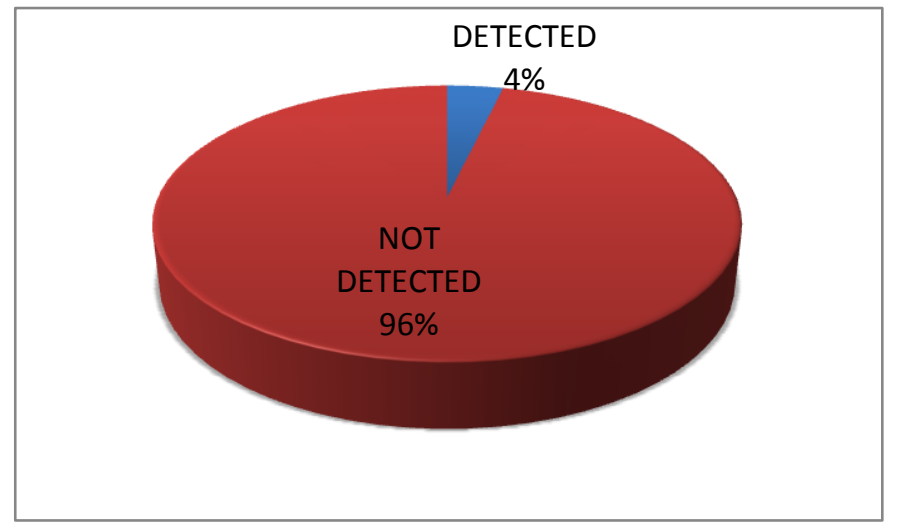

Rifampicin resistance was detected in 2 out of 54 smear negative MTB detected cases which is similar to Sowjanya et al (3.6\%)

\section{Discussion}

In present study, out of 140 cases, 98 (70\%) were males and rest $42(14 \%)$ were females. Male predominance was in same line with similar with Mohanty et al (23/71) and Ishwarappagol et al (28/80) studies. In this study population, 51- 60yrs age group was commonly affected. Cough with expectoration followed by breathlessness being the predominant features in this study as same as France Le Palud et al (2014) and Mishra et al study. $^{20,21} 32.9 \%$ of study population were diabetics; out of them, 17 were detected to PTB. Among these diabetic PTB patients, 10 (59.1\%) have lower zone involvement as same as Pradeep Ishwarappagol et al study. ${ }^{18}$ Only $5(3.6 \%)$ of study population were HIV reactive. Rest of 135(96.4\%) were HIV Non-reactive. Out of 5 PLHIV, only 2 of them were reported as MTB detected.

Smoking and alcohol being a major risk factor contributes about $57.1 \%(80)$ and $37.9 \%$ (53) for causing pulmonary tuberculosis in this study population respectively. Among study population, $37(26.4 \%)$ have positive contact history. $27.1 \%$ (38) of study population have past history of pulmonary tuberculosis. Out of these, 18 were defaulter and rest of the 20 completed the treatment. 35\% (49) of study population are in low BMI group. Infiltrates (36) are the most common radiological lesion in this study similar to poojan shrestha et $\mathrm{al}^{22}$ (2015) followed by fibro cavity and consolidation. In CBNAAT positive cases, fibro cavity and infiltrate reported as predominant finding whereas in CBNAAT negative, consolidation is the more frequently reported correlating with Poojan Shretha et al.

This study consists of 140 smear negative presumptive pulmonary tuberculosis cases. CBNAAT was conducted. Out of these 140 cases, MTB was detected in 54 cases $(38.6 \%)$ and in rest of the 86 cases MTB not detected. Among 54 cases, 2 were rifampicin resistance and rest of them were rifampicin sensitive. Among 86 MTB not detected cases, $2.3 \%$ (2/86) of them were started on ATT drugs based on the clinical features and chest X-ray finding. The sensitivity and specificity in smear negative sputum sample correlates with other studies from $57 \%-75 \%$ and $97-100 \%$ respectively. ${ }^{23-26}$ In this study MTB was detected at medium level in 24 cases $(17.1 \%)$ followed by low level in 17(12.1\%) of them.

Rifampicin resistance has been reported in 2 out of 54 CBNAAT positive cases, which is similar to Sharma et al study. These 2 rifampicin resistance 
was diagnosed in newly detected pulmonary tuberculosis patients. Interestingly both of them have lower zone involvement. Drug surveillance was performed in many countries indicating the prevalence of MDR TB in 2-3\% of newly diagnosed pulmonary tuberculosis patient and $17 \%$ of retreated cases. This study shows the prevalence of $3.7 \%$ MDR-TB in new case which correlates with state-wide study done at Gujarat by ramachandranetal. ${ }^{27}$
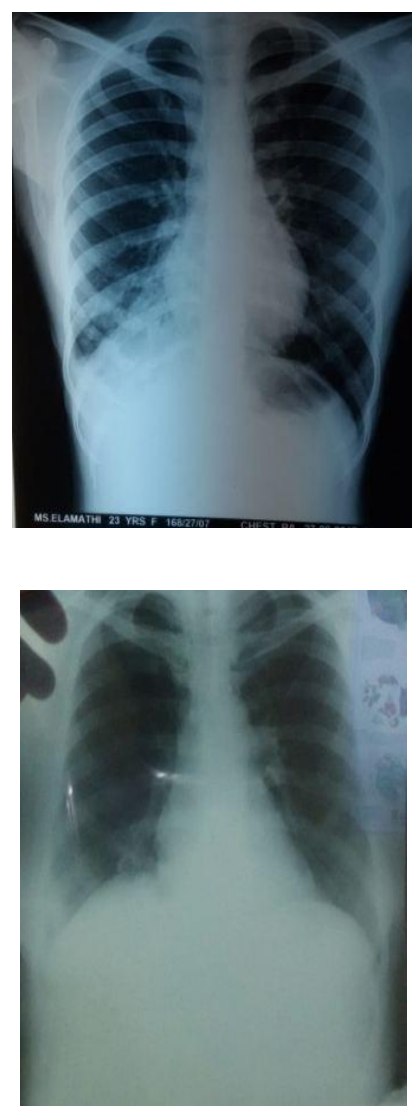

Chest $x$-ray of rifampicin resistance in new smear negative cbnaat positive pulmonary tuberculosis

\section{Conclusion}

In this study, we concluded that CBNAAT is more superior to sputum microscopy by detecting additional pulmonary tuberculosis cases which are missed by sputum AFB stain. CBNAAT's additional benefit of detecting rifampicin resistance gives confidence to many clinicians by starting appropriate treatment on the same day and reducing the risk of transmission of MDR TB. Various studies prove that MDR TB is common among retreated cases. But this study proves the possibilities of MDR TB in new smear negative CBNAAT positive pulmonary tuberculosis. We concluded that all sputum AFB negative presumptive pulmonary tuberculosis patients should undergo novel technique like CBNAAT and LPA to diagnose the undetected TB and DR TB. CBNAAT negative does not exclude the diagnosis of tuberculosis.

\section{References}

1. Helb D, jones $\mathrm{M}$,Story $\mathrm{E}$, Boehme C, Wallace E, H K , Kop J, Owens MR, Rodgers R, Banada $\mathrm{P}$, et al. Rapid detection of mycobacterium tuberculosis and rifampicin resistance by use of on demand, near patient technology. J ClinMicrobiol 2010; 48: 229 - 237.

2. Boehme CC, Nabeta P, Hillmann D, NicolMP, Shenai S, Krapp F , Allen J,Tahirli R, Blakemore R, rustomjee R, et al. Rapid molecular detection of tuberculosis and rifampicin resistance .N Eng J Med 2010: 363: 1005- 1015.

3. Van Rie A, page - Shipp L, Scott L, Sanne I, tevens W. Xpert MTB/RIF for pint of care diagnosis of TB in high - HIV burden, resource limited countries: hype or hope? Expert Rev MolDiagn 2010;10: 937 $-946$.

4. Automated real time nucleic acid amplification technology for rapid and simultaneous detection of tuberculosis and rifampicin resistance: Xpert MTB/RIF system for the diagnosis of pulmonary and extra pulmonary tuberculosis TB in adults and children : policy update. Geneva: World Health Organization, 2013. http://www.who. ot/tb/laboratory/policy_statements/en/)

5. Lawn SD, Nicol MP. Xpert MTB/RIF assay: development, evaluation and implementation of a new rapid molecular diagnostic for tuberculosis and rifampicin 
resistance. Future microbial 2011;6(9):1067-82

6. Guidance document for use of Catridge Based Nucleic Acid Amplification Test (CB-NAAT) under Revised National TB Control Programme (RNTCP) issued central TB division, directorate general of health services September 2013.

7. World Health Organization. Global tuberculosis report Geneva: WHO, 2016.

8. http://www.tbfacts.org

9. World Health Organization. Fluorescent light - emitting diode (LED) microscopy for diagnosis of tuberculosis. Policy statement.

WHO/HTM/TB/2011.8.Geneva,

Switzerland: WHO, 2011.

10. Stop TB Partnership and World Health Organization. New Laboratory Diagnostic Tools for TB control. Geneva, World Health Organization, 2008.

11. Manna N, Giri K, Mundle M et al. Drug resistance pattern, related socio demographic factor and prevalence practice among MDR-TB patients: An experience from a tertiary care setting. JDMS: 2014; 3 (9); 16-21.

12. Foundation for Innovative New Diagnostics. Performance of Xpert MTB/RIF version G4assay.Geneva: Foundation for innovative new diagnostics;2011.http://www.stoptb.org/w $\mathrm{g} / \mathrm{gli} /$ assets/documents/map/findg4catridge .pdf.Accessed 22 Nov 2016.

13. Boehme CC, Nabeta P, Nicol et al. Feasibility, diagnostic accuracy, effectiveness of decentralized use of the Xpert MTB/RIF test for diagnosis of tuberculosis and multidrug resistance: Lancet a muticentric implementation study. 2011:377 (9776): 1495-1505.

14. Chakraborty S, Sen MK, Tyagi JS. Diagnosis of extra pulmonary tuberculosis by smear, culture, and PCR using sample processing technology. J Clin
Microbiol.2005; 43:4357 - 62 PMID: 16145077.

15. Steingart KR, SchillerI, Horne DJ, PaiM, Boehme CC, Dendukuri N. Xpert ${ }^{\circledR}$ MTB/RIF assay for pulmonary tuberculosis and rifampicin resistance in adults(review). Cochrane Database Syst. Rev1:CD009593. http://dx. doi.org/ $10.1002 / 14651858$.

CD009593.pub2.doi:10.1002/14651858.C D009593 pub3PID:24448973.

16. Blackmore R, Story E, Helb D, et al. Evaluation of the analytical performance of the Xpert MTB/RIF assay. J Clin Microbial 2010;48 (7):2495-2501.

17. Sowjanya DS, Behera G, Reddy VVR, et al: Novel diagnostic tool for rapid and specific detection of Mycobacterium tuberculosis in pulmonary samples. IJHRMIMS 2014:28-31.

18. Rao KNM ,Vinod K, Ishwarappagol P, et al. Efficacy of CBNAAT in detecting sputum negative pulmonary tuberculosis.

19. Weyer k, Mirzayev F, Milgliori GB, Gemert WV, D'Ambrosio L, ZignolM.Rapid molecular TB diagnosis :evidence, policy making and global implementation of Xpert MTB/RIF.ERJ.2013;42(1): 252-71.

20. Pierre Lee Palud, Vincent Cattoir, Bridgitte Malbruny, Romain Magnier, Karine Campbell, Youssef Oilkhouir1, et al. Retrospective observational study of diagnostic accuracy of the Xpert MTB/RIF assay on the fiberoptic bronchoscopy sampling for early diagnosis of smear negative or smear scare patients with suspected tuberculosis. BMC Pulmonary Medicine 014;14:137.

21. Avashia S, Choubey S,Mishra S et al.To study the usefulness of CBNAAT (Catridge Based Nucleic Acid Amplification Test) in BAL (bronchoalveolar lavage) samples in the diagnosis of smear negative/non sputum 
producing patients with suspected tuberculosis.

22. Poojan Shrestha et al. The Application of Gene Xpert MTB/RIF for Smear Negative TB diagnosis as a fee paying service at a South Asian General Hospital.

23. NorinJ (May 2015) A retrospective evaluation of diagnostic accuracy of Xpert ${ }^{\circledR} \quad$ MTB/RIF assay, used for detection of Mycobacterium tuberculosis in Greece.http://www.divportal.se/smash/get/diva2:825390/FULLT EXT01.pdf.

24. Zeka AN, Tasbakan S, Cavusoglu C. Evaluation of the gene XpertMTB/RIF for rapid diagnosis of Tuberculosis and detection of rifampicin resistance in pulmonary and extra pulmonary tuberculosis. JClinMicrobiol. 2011; 49: 4138-41.

25. Geleta DA, Megerssa YC, Gudeta AN, et al. Xpert MTB/RIF assay for diagnosis of pulmonary tuberculosis in sputum specimen in remote care facility. BMC Microbiology 2015;15: 220.

26. Sharma SK, Kohli M, Yadav RN, et al. Evaluating the diagnostic accuracy of Xpert with ZN stain and culture in samples of suspected pulmonary tuberculosis. Journal of Clinical and Diagnostic Research 2016; 10(5):DC09-DC12.

27. Ramachandran R, Nalini S, Chandrasekar V, Dave PV, Sanghavi AS, Wares, et al. Surveillance of drug resistance tuberculosis in the state of Gujarat, India. Int J Tuberc Lung Dis 2009; 13(9): 115460. 